\title{
Nuevos datos paleontológicos del Pleistoceno en el Valle del Manzanares (Madrid, España): Los micromamíferos del yacimiento del Arenero de Arriaga
}

\author{
New paleontological data from the Pleistocene Manzanares Valley \\ (Madrid, Spain): The micromammals from the Arenero de Arriaga site
}

\author{
Carmen Sesé ${ }^{1}$ y Nieves López Martínez (†)
}

\begin{abstract}
RESUMEN
Se describe la asociación de micromamíferos del yacimiento achelense del Arenero de Arriaga del valle del Manzanares constituida por los soricomorfos: Crocidura sp. y Talpa sp., los roedores: Eliomys quercinus quercinus, Apodemus sp., Microtus brecciensis, Microtus arvalis, Microtus duodecimcostatus y Arvicola aff. sapidus, y el lagomorfo: Oryctolagus cuniculus. La asociación faunística y el estadio evolutivo de Microtus brecciensis y Arvicola aff. sapidus, indican una edad del final del Pleistoceno Medio. La asociación de micromamíferos sugiere la existencia de varios biotopos representados: ripícola, praderas húmedas y secas, y bosque, y un clima templado similar al actual de la Meseta.
\end{abstract}

Palabras clave: Soricomorfos, Roedores, Lagomorfos, Pleistoceno Medio final, Paleoecología, Paleoclimatología, Valle del Manzanares, Madrid.

\begin{abstract}
The micromammals from the archaeological site of the Arenero de Arriaga from the Manzanares Valley are here described. They are the Soricomorpha: Crocidura sp. and Talpa sp., the Rodentia: Eliomys quercinus quercinus, Apodemus sp., Microtus brecciensis, Microtus arvalis, Microtus duodecimcostatus and Arvicola aff. sapidus, and the Lagomorpha: Oryctolagus cuniculus. The faunal association and the evolutionary state of Microtus brecciensis and Arvicola aff. sapidus, suggest an age of the end of the Middle Pleistocene. It also indicates the existence of different biotopes: riparian, moist and dry meadows, and forest, and a temperate climate similar to the present-day climate of the Meseta.
\end{abstract}

Keywords: Soricomorpha, Rodentia, Lagomorpha, End of the Middle Pleistocene, Palaeoecology, Palaeoclimatology, Manzanares Valley, Madrid.

\section{Introducción y antecedentes}

El yacimiento del Arenero de Arriaga está situado en el término municipal de Rivas-Vaciamadrid, al sur de Madrid capital, en la margen derecha del río Manzanares, en la llamada Terraza Compleja de Butarque por Goy et al. (1989), que otros autores denominan Terraza Compleja del Manzanares (Silva, 2003; Silva et al., 2011 y 2012).
La situación del yacimiento, la estratigrafía y una lista faunística preliminar de la microfauna: herpetofauna y micromamíferos (determinados respectivamente por B. Sanchiz y N. López) procedentes del lavado de una pequeña cantidad de sedimento, fueron publicadas por Gamazo (1982: p. 55-56). Posteriormente Rus \& Vega (1984) dieron a conocer la estratigrafía y los hallazgos arqueológicos y de algunos macromamíferos, y Rus (1989) los resul-

\footnotetext{
${ }^{1}$ Dpto. Paleobiología, Museo Nacional de Ciencias Naturales, C.S.I.C., J. Gutiérrez Abascal 2, 28006 Madrid, España. Email: c.sese@mncn.csic.es

(†) Nieves López lamentablemente murió durante la elaboración de este manuscrito. La co-autora le dedica este trabajo en su memoria.
} 
tados arqueológicos y paleontológicos preliminares de micromamíferos y macromamíferos (determinados respectivamente por N. López y C. Sesé, y E. Soto en: Rus, 1989: p. 34), procedentes de las excavaciones realizadas de forma intensiva en el Arenero de Arriaga entre 1981 y 1983. Con anterioridad a estas dos últimas publicaciones científicas, en un artículo de divulgación sobre el Paleolítico en el Manzanares, Rus (1983) describió los hallazgos arqueológicos y de macromamíferos más importantes realizados en dicho yacimiento.

Paralelamente a estas últimas excavaciones, bajo la dirección de Inmaculada Rus y la supervisión geológica de Manuel Hoyos, se lavó gran cantidad de sedimento del yacimiento del que procede el material de micromamíferos objeto del presente trabajo.

Los restos de micromamíferos provienen de los limos arcillosos de color verde oscuro, de llanura de inundación, de la unidad denominada Arriaga I. Rus (1989) realizó la estratigrafía detallada de las tres unidades diferenciadas en el yacimiento: Arriaga I, II a y b y III, actualizada recientemente por Silva et al. (2011 y 2012). Arriaga I es el nivel que ha proporcionado los restos de micromamíferos, herpetofauna, peces, gasterópodos y también industria lítica; Arriaga II: en el nivel Arriaga IIa se encontró gran parte de un esqueleto de Elephas (Palaeoloxodon) antiquus asociado a un conjunto lítico, y en Arriaga IIb industria lítica del Achelense Superior asociada a numerosos restos de diversos taxones de macromamíferos; y finalmente en Arriaga III se recuperaron restos de una defensa de Elephas (Palaeoloxodon) antiquus y numerosos gasterópodos acuáticos (Rus \& Vega, 1984; Rus, 1989; Silva, 2003; Silva et al., 2011 y 2012).

Sesé \& Soto (2000 y 2002 a y b), basándose en la asociación de micro y macromamíferos, asignaron a este yacimiento una edad del final del Pleistoceno Medio o tránsito al Pleistoceno Superior.

Trabajos recientes realizados en la terraza donde se ubica el yacimiento (Pérez-González et al., 2008; Silva et al., 2008; Domínguez Alonso et al., 2009), y las últimas dataciones por termoluminiscencia (TL), de la base al techo en la secuencia en la que está el yacimiento del Arenero de Arriaga (Silva et al., 2011 y 2012), han proporcionado unos valores entre fechas anteriores a 134-133 ka BP para Arriaga I (nivel del que proceden los micromamíferos) y Arriaga II, hasta $96 \pm 21 / 13 \mathrm{ka} \mathrm{BP}$ para Arriaga III. Es decir que las dataciones obtenidas por dichos autores (o.c.) concuerdan con la edad propuesta biostratigráficamente por la fauna con anterioridad por Sesé \& Soto (2000 y 2002 a y b).
Además de los micromamíferos que se estudian en este trabajo, el resto de la fauna del yacimiento de Arriaga, procedente de las excavaciones de I. Rus, es la siguiente: Arriaga II: Elephas (Palaeoloxodon) antiquus, Bos primigenius, Equus caballus, Cervus elaphus y Dicerorhinus hemitoechus; Arriaga III: Elephas (Palaeoloxodon) antiquus (Rus, 1989; Sesé \& Soto, 2000 y 2002 a y b).

La herpetofauna identificada en el yacimiento corresponde a la fauna recuperada por Mercedes Gamazo (Sanchiz com. pers.) y es la siguiente: Pelobates cultripes, Bufo bufo, Rana perezi, Lacerta lepida, Lacertidae talla pequeña (dos formas) (Gamazo, 1982; Sanchiz 1991). El nivel en el que Gamazo (1982) recuperó la herpetofauna y los micromamíferos, parece corresponder al nivel de Arriaga I del que, según Rus (1989) se han recuperado prácticamente todos los restos de microfauna: peces, anfibios, reptiles y micromamíferos, así como gasterópodos.

El yacimiento tiene también una gran importancia arqueológica al haber proporcionado una rica industria del Achelense Superior (Rus \& Vega, 1984; Rus \& Enamorado, 1991).

El presente estudio de los micromamíferos del Arenero de Arriaga tiene el interés, por una parte, de completar la información paleontológica de un yacimiento que de forma excepcional reúne restos de la actividad humana y restos fósiles de una rica y variada fauna de grandes y pequeños mamíferos y otros microvertebrados como se ha dicho, y, por otra, completar los datos paleontológicos de micromamíferos del sur de Madrid. En esta zona, hasta finales del siglo pasado, además de los mencionados datos preliminares de micromamíferos del Arenero de Arriaga (Gamazo, 1982; Rus, 1989), solo se conocían los micromamíferos del yacimiento arqueológico y paleontológico del Pleistoceno Medio avanzado de Áridos-l en las terrazas del valle del Jarama (López Martínez, 1980 a y b), situado en la unidad basal de Arganda I de las cuatro unidades definidas en la cantera de Áridos (Pérez González, 1971 y 1980). En la Terraza Compleja de Arganda (Pérez González, 1971), las diversas dataciones por racemización de aminoácidos realizadas recientemente proporcionan unas fechas para la unidad de Arganda I en la que se ubica el yacimiento de Áridos-1 entre 335 ka BP y 370 ka BP, que se corresponden con el tramo final del MIS 11 (Panera et al., 2011). Estos datos concuerdan con la biocronología basada en la asociación de micromamíferos propuesta anteriormente por López Martínez (1980 a), 
que precisó la edad de la asociación faunística, dentro del Pleistoceno Medio, en: "el interglaciar Mindel-Riss (-300.000-350.000 años)" (López Martínez, 1980 a: p. 194).

Los citados yacimientos de Áridos-1 y Arenero de Arriaga fueron los primeros del Cuaternario de Madrid en los que durante finales de los años $70 \mathrm{y}$ principios de los 80 del siglo pasado, N. López primero y las autoras de este trabajo después, respectivamente, aplicaron de forma intensiva los procesos de lavado y tamizado del sedimento que son imprescindibles para la recuperación de restos de micromamíferos y en general de microfauna. Ello explica la falta de datos sobre estos grupos que había hasta entonces, como se pone de manifiesto en las síntesis paleontológicas de Madrid realizadas por Sesé \& Soto (2000, 2002 a y b), y arqueológica y paleontológica de Baena et al. (2002) sobre los numerosos yacimientos pleistocenos que hay en el sur de Madrid, de los que hasta entonces solo había datos de macromamíferos.

Casi veinte años después de las excavaciones y trabajos sistemáticos realizados sobre micromamíferos en Áridos-1 y en el Arenero de Arriaga, en la pasada década de este siglo, y gracias a diversos planes de actuación propiciados por la Comunidad de Madrid, se reactivaron las prospecciones y excavaciones en el sur de Madrid dando lugar a numerosos hallazgos de yacimientos arqueológicos y paleontológicos del Pleistoceno Medio, Pleistoceno Superior y Holoceno. En el valle del Jarama cabe destacar los yacimientos con micromamíferos de Valdocarros del Pleistoceno Medio avanzado pero no final, situado en la unidad de Arganda II, del que se dispone de diversas dataciones por racemización de aminoácidos entre 255 ka BP y $269 \mathrm{ka}$ BP, que se corresponderían con el inicio del MIS 7, y HAT del Pleistoceno Superior, situado en la unidad de Arganda IV y datado por TL en 74+16/-12,1 ka BP, lo que le sitúa a finales del MIS 5 (Panera et al., 2005 y 2011; Sesé et al., 2011 a). En el valle del Manzanares se encuentran los yacimientos del Pleistoceno Medio de TAFESA (antigua TRANSFESA), en el que, a diferencia de los demás yacimientos mencionados, la escasez de restos de micromamíferos no permiten realizar precisiones biostratigráficas en base a este grupo, pero sí a partir de los macromamíferos (Baena et al., 2010; Mazo, 2010; Sesé, 2010), y el del Pleistoceno Superior de PRERESA con una datación obtenida mediante luminiscencia (OSL) de $84 \pm 5,6 \mathrm{ka} \mathrm{BP}$ (Sesé et al., 2011 b).
Hay que destacar que todas las dataciones absolutas realizadas recientemente por diversos métodos en los mencionados yacimientos paleontológicos del sur de Madrid se corresponden perfectamente con las edades biocronólogicas basadas en micromamíferos propuestas por López Martínez (1980 a) y Sesé et al. (2011 a y b).

\section{Metodología}

Se lavaron con agua unos $3.500 \mathrm{~kg}$ de sedimento, haciéndolo pasar por una serie de tamices de los que el más inferior era de luz de malla de $0,5 \mathrm{~mm}$.

Los dientes se han medido con una lupa que lleva incorporado un micrómetro de $0,025 \mathrm{~mm}$ de precisión. Las medidas, tomadas en la superficie oclusal de los dientes orientados en su correspondiente posición anatómica, se expresan en milímetros (mm) y corresponden a la longitud (L) y anchura (A) máximas. Las medidas aproximadas de los dientes fragmentados se dan entre paréntesis. La nomenclatura utilizada para los dientes es según Van der Meulen (1973) para los arvicolinos y López Martínez (1980 a) para el resto de los grupos. Los dibujos han sido realizados utilizando una cámara clara acoplada a un microscopio Nikon SMZ-IO con 40 aumentos. Para comparar la abundancia relativa de los taxones se ha utilizado el NMI (Número Mínimo de Individuos) calculado sobre la pieza dentaria (esquelética en el caso del topo) taxonómicamente identificable y más abundante teniendo en cuenta el número máximo de piezas derechas o izquierdas.

El material dentario y esquelético se ha comparado con formas actuales de micromamíferos de las colecciones del Museo Nacional de Ciencias Naturales (MNCN).

\section{Paleontologia sistemática}

Orden Soricomorpha GREGORY, 1910

Familia Soricidae FISCHER, 1814

Subfamilia Crocidurinae MILNE-EDWARDS, 1872

Crocidura sp. - Musaraña

(Fig. 1: 1)

Material: 1 fr. de mandíbula der. con $\mathrm{M}_{1}-\mathrm{M}_{2}-\mathrm{M}_{3} ; \mathrm{NMI}=1$

Medidas: $\mathrm{L} \mathrm{M}_{1}-\mathrm{M}_{3}=3,52 ; \mathrm{M}_{1}: \mathrm{L}=1,34 ; \mathrm{A}=0,88 ; \mathrm{M}_{2}: \mathrm{L}=$ 1,$27 ; \mathrm{A}=0,83 ; \mathrm{M}_{3}: \mathrm{L}=1,05 ; \mathrm{A}=1,61$.

Descripción y discusión: Los dientes no presentan pigmentación sino el color blanquecino de los crocidurinos. El $\mathrm{M}_{3}$ presenta los rasgos característicos de este grupo con el talónido reducido a una sola cresta y sin valles (López Martínez, 1980 a). Su talla, claramente mayor que la de las poblaciones actuales del género Suncus, en el que además la reducción del talónido del $\mathrm{M}_{3}$ es, por comparación, extremo, le diferencian de dicho género. Su morfología, especialmente la del talónido del $\mathrm{M}_{3}$, es sin embargo similar a la del género 
Crocidura. Su talla es algo menor que la de las poblaciones actuales de las colecciones del MNCN de Crocidura russula (HERMANN, 1780) y datos de Sesé et al. (2011 b), menor también que la de esta especie del yacimiento de PRERESA (Sesé et al., 2011 b) y ligeramente superior a la de Crocidura aff. russula del yacimiento de Áridos-1 (López Martínez, 1980 a). Dado que en la mandíbula del Arenero de Arriaga no se conserva la rama ascendente ni el cóndilo (parte anatómica que según López Martínez, 1980 a, presenta caracteres que permiten distinguir varias especies del género Crocidura), ni hay otro tipo de material dentario como los dientes superiores que según dicha autora (o.c.) presentan caracteres de interés taxonómico para distinguir especies, no se puede realizar su atribución específica.

En la Península Ibérica se registran Crocidura russula desde el Pleistoceno Medio y Crocidura suaveolens (PALLAS, 1811) desde el Pleistoceno Superior (Sesé, 1994; Arribas, 2004). Actualmente la primera especie tiene una amplia distribución por toda la Península Ibérica. mientras que la segunda tiene una distribución escasa y fragmentada (Palomo \& Gisbert, 2002).

Familia Talpidae FISCHER, 1814

Subfamilia Talpinae FISCHER, 1814

Talpa sp. - Topo

Material: 1 radio der.; 1 radio iz.; 1 fr. de falange. $\mathrm{NMI} \mathrm{=} 1$

Descripción y discusión: En una comparación con material actual, estas piezas esqueléticas presentan la talla y características propias del género Talpa, cuya particular morfología del esqueleto apendicular, debido a sus hábitos cavadores y vida subterránea, presenta unas modificaciones que le diferencian claramente de los demás taxones de soricomorfos y micromamíferos en general. No obstante, al no haberse hallado otro material como el dentario u otras piezas del esqueleto apendicular, no se puede realizar su atribución específica.

$\mathrm{Su}$ registro en el Arenero de Arriaga es la primera cita de este taxón en el sur de Madrid. En la Península Ibérica, Talpa europaea LINNAEUS, 1758 se registra desde el Pleistoceno Medio y Talpa occidentalis CABRERA, 1907 posiblemente desde el Pleistoceno Superior (Sesé, 1994 y 2005; Arribas 2004). Arsuaga et al. (2010) citan ambas especies en el yacimiento del Pleistoceno Superior del norte de Madrid de la Cueva del Camino (Pinilla del Valle). Actualmente Talpa europaea está en el tercio norte peninsular, mientras que Talpa occidentalis, endémica de la Península Ibérica, se distribuye sobre todo y de forma continua por la región noroccidental, pero falta en los Pirineos y valle del Ebro y en el resto de la península se encuentra en zonas montañosas (Palomo y Gisbert, 2002).

Orden Rodentia BODWICH, 1821

Familia Gliridae MUIRHEAD, 1819

Eliomys quercinus quercinus (LINNAEUS, 1766)

- Lirón careto

(Fig. 1: 2 y 3)

Material: $1 \mathrm{P}_{4} ; 2 \mathrm{M}_{1} ; 1 \mathrm{M}_{3} ; 1 \mathrm{D}^{4} ; 3 \mathrm{P}^{4} ; 2 \mathrm{M}^{1-2} ; 1$ fr. $\mathrm{M}^{1-2}$; $\mathrm{NMI}=2$
Medidas:

\begin{tabular}{|c|c|c|c|c|c|c|c|}
\hline \multirow{2}{*}{ DIENTE } & \multirow{2}{*}{$\mathrm{N}$} & \multicolumn{3}{|c|}{ LONGITUD } & \multicolumn{3}{c|}{ ANCHURA } \\
\cline { 3 - 8 } & & MÍN. & MED. & MÁX. & MÍN. & MED. & MÁX. \\
\hline $\mathrm{P}_{4}$ & $1 / 1$ & ----- & 1,45 & ---- & ---- & 1,31 & ----- \\
\hline $\mathrm{M}_{1}$ & $2 / 2$ & 1,58 & 1,59 & 1,60 & 1,70 & 1,74 & 1,79 \\
\hline $\mathrm{M}_{3}$ & $1 / 1$ & ----- & 1,47 & ---- & ---- & 1,41 & ----- \\
\hline $\mathrm{D}^{4}$ & $1 / 1$ & ----- & 1,08 & ----- & ---- & 1,18 & ----- \\
\hline $\mathrm{P}^{4}$ & $3 / 3$ & 1,15 & 1,17 & 1,19 & 1,52 & 1,65 & 1,73 \\
\hline $\mathrm{M}^{1-2}$ & $2 / 2$ & 1,38 & 1,44 & 1,51 & 1,70 & 1,81 & 1,92 \\
\hline
\end{tabular}

Descripción: La morfología del $\mathrm{P}_{4}$ es simple, con las tres cúspides principales, anterocónido, entocónido e hipocónido, muy notables, y sin crestas intermedias entre anterolófido y posterolófído. $\mathrm{M}_{1}$ y $\mathrm{M}_{3}$ tienen una morfología similar a la de Eliomys quercinus actual, sin crestas accesorias entre las crestas principales. En el $\mathrm{M}_{1}$ hay un centrolófido débil y corto; en el $\mathrm{M}_{3}$ no existe centrolófido. En $\mathrm{D}^{4}$ y $\mathrm{P}^{4}$ hay anterolofo y están presentes las demás crestas principales que existen en los molares superiores. En dos de los tres $\mathrm{M}^{1-2}$ hay dos centrolofos de igual desarrollo, unidos respectivamente a paracono y metacono, y en el otro ejemplar hay un solo centrolofo: el endolofo es continuo y no hay crestas accesorias entre las crestas principales.

Discusión: Tanto por su morfología como por su talla este material pertenece a la especie actual Eliomys quercinus. La simplicidad de la morfología de los dientes del Arenero de Arriaga, sin crestas accesorias entre las crestas principales en los molares superiores e inferiores, le asemeja a la subespecie Eliomys quercinus quercinus. La ausencia en los molares de crestas accesorias y de mesolófido en el $\mathrm{P}_{4}$ diferencian a la población del Arenero de Arriaga de Eliomys quercinus helleri JANOSSY, 1962, descrito por Chaline (1972) en los yacimientos franceses del Pleistoceno Medio de Saint-Stève-Janson y Orgnac 3, y de Eliomys quercinus granatensis LÓPEZ MARTÍNEZ y RUIZ BUSTOS, 1977 del yacimiento del final del Pleistoceno Medio de Cueva del Agua (López Martínez \& Ruiz Bustos, 1977) que sí las presentan. Eliomys quercinus helleri tiene, además, una talla menor que el glírido del Arenero de Arriaga.

Eliomys quercinus en España se registra desde el final del Pleistoceno Inferior hasta la actualidad (Sesé, 1994; Sesé \& Sevilla, 1996). Está presente en casi todos los yacimientos del sur de Madrid: Áridos-1 (López Martínez, 1980 a), Valdocarros y HAT (Sesé et al., 2011 a) y PRERESA (Sesé et al., 2011 b). Es muy común actualmente en toda la Península Ibérica e Islas Baleares excepto en Ibiza (Blanco, 1998; Palomo \& Gisbert, 2002). Según dichos autores (o.c.) hay dos subespecies en la Península Ibérica, Eliomys quercinus lusitanicus en la región suroccidental y Eliomys quercinus quercinus, de menor tamaño que la anterior, en el resto de la Península.

Familia Muridae ILLIGER, 1811

Apodemus sp. - Ratón

(Fig. 1: 4 y 5)

Material: 1 fr. de mandíbula der. con $\mathrm{I}_{-} \mathrm{M}_{1}-\mathrm{M}_{2} ; 1$ fr. de mandíbula izq. con $\mathrm{M}_{1} ; 1$ fr. $\mathrm{M}_{2} ; 4 \mathrm{M}_{3} ; 1$ fr. $\mathrm{M}^{1} ; 1 \mathrm{M}^{2} ; \mathrm{NMI}=2$ 
Medidas:

\begin{tabular}{|c|c|c|c|c|c|c|c|}
\hline \multirow{2}{*}{ DIENTE } & \multirow{2}{*}{$\mathrm{N}$} & \multicolumn{3}{|c|}{ LONGITUD } & \multicolumn{3}{c|}{ ANCHURA } \\
\cline { 3 - 8 } & & MÍN. & MED. & MÁX. & MÍN. & MED. & MÁX. \\
\hline $\mathrm{M}_{1}$ & $2 / 2$ & 1,98 & 1,98 & 1,99 & 1,20 & 1,21 & 1,23 \\
\hline $\mathrm{M}_{2}$ & $1 / 1$ & ---- & 1,30 & ---- & ---- & 1,19 & ---- \\
\hline $\mathrm{M}_{3}$ & $4 / 4$ & 0,89 & 0,98 & 1.10 & 0,83 & 0,89 & 0,98 \\
\hline $\mathrm{M}^{2}$ & $1 / 1$ & ---- & 1,39 & ---- & ---- & 1,19 & ---- \\
\hline
\end{tabular}

Descripción: En el $\mathrm{M}_{1}$ el tubérculo medio anterior (tma) es relativamente grande aunque de menor tamaño que los tubérculos anteriores; el margen cingular labial está bien desarrollado y aparecen sobre el mismo de tres a cuatro cúspides accesorias bien diferenciadas así como una cúspide accesoria sobre el cíngulo posterior. En el $\mathrm{M}^{2}$ el T9 no está reducido siendo de un tamaño similar al T6.

Discusión: El múrido del Arenero de Arriaga es de talla y morfología similares a las de Apodemus sylvaticus (LINNAEUS, 1758) y Apodemus flavicollis (MELCHIOR, 1834). Pasquier (1974) y Michaux \& Pasquier (1974) diferencian ambas especies en base principalmente a varios caracteres del $\mathrm{M}^{2}$. El único $\mathrm{M}^{2}$ disponible del Arenero de Arriaga tiene un T9 no reducido, lo que le asemeja a Apodemus sylvaticus, sin embargo, la relación L/A, con un valor de 1,15, le aproxima a Apodemus flavicollis. Estos caracteres de diferenciación son válidos en poblaciones relativamente numerosas. La determinación específica del múrido del Arenero de Arriaga no es por tanto posible debido a la escasez de material.

Apodemus sylvaticus tiene actualmente una amplia distribución por toda la Península Ibérica, mientras que Apodemus flavicollis solo está en el tercio norte peninsular (Blanco, 1998; Palomo \& Gisbert, 2002).

Familia Cricetidae FISCHER, 1817

Subfamilia Arvicolinae GRAY, 1821

Microtus brecciensis (GIEBEL, 1847) - Topillo de las brechas

(Fig. 1: 6)

Material: 1 fr. de mandíbula der. con $\mathrm{M}_{1} ; 1 \mathrm{M}_{1} ; 1$ fr. $\mathrm{M}_{1}$ juvenil; $2 \mathrm{M}^{3}$; $\mathrm{NMI}=2$

Medidas: $2 \mathrm{M}_{1} ; \mathrm{L}=3,22-3,23 ; \mathrm{A}=1,30-(1,35) ; 2 \mathrm{M}^{3}: \mathrm{L}=$ $2,12-2,15 ; \mathrm{A}=1,09-1,12$

Descripción: Hay dos $\mathrm{M}_{1}$ de individuos adultos que presentan la morfología característica de Microtus brecciensis: molares arrizodontos, con cemento en los ángulos entrantes, esmalte más grueso en el borde anterior de los triángulos y ausente en el borde antero-labial de la cúspide anterior y bordes lingual y labial del lóbulo posterior, y los cinco primeros triángulos cerrados, así como los siguientes caracteres: en el complejo anterocónido los ángulos entrantes LRA 4 y BRA 3 son alternos, el LRA 5 está relativamente desarrollado y no hay esbozo de BRA 4. Esta morfología corresponde al morfotipo "nivaloide" típico de la especie, morfotipo $\mathrm{n}^{\circ} 6$ de Microtus brecciensis de Áridos-1 que es el más frecuente en dicha
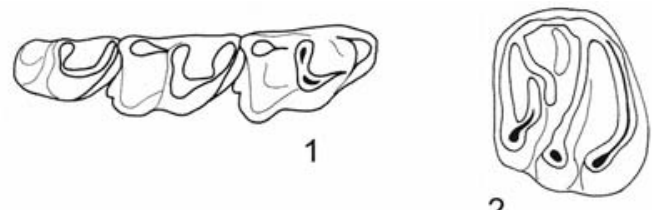

2
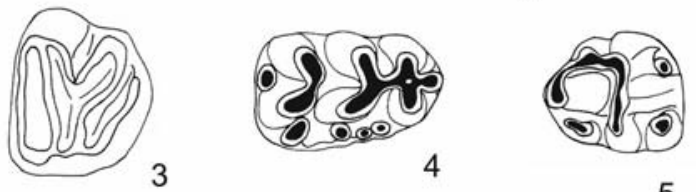

5
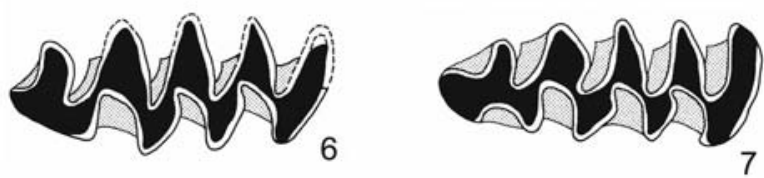

7
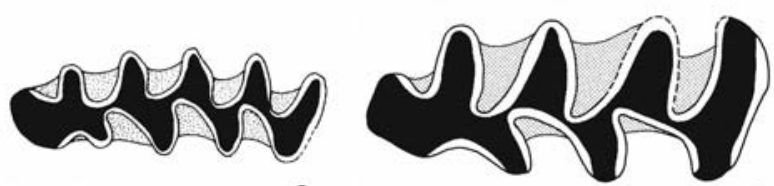

8

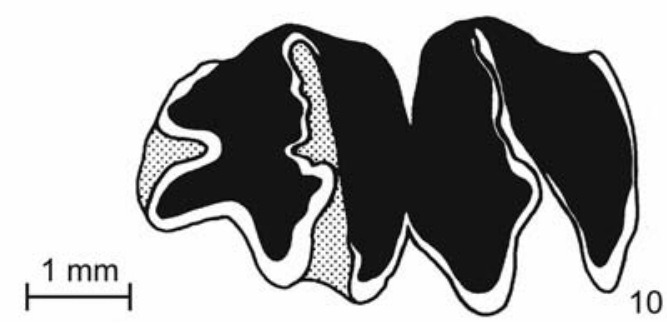

Fig. 1.-Dibujos de los dientes de Micromamíferos del yacimiento del Arenero de Arriaga en vista oclusal: Crocidura sp.: 1: Serie dentaria inferior derecha: $\mathrm{M}_{1}-\mathrm{M}_{2}-\mathrm{M}_{3}$; Eliomys quercinus quercinus: 2: $\mathrm{M}_{1}$ izq.; 3: $\mathrm{M}^{1-2}$ izq.; Apodemus sp.: 4: $\mathrm{M}_{1}$ der.; 5: $\mathrm{M}^{2}$ der.; Microtus brecciensis: 6: $\mathrm{M}_{1}$ izq.; Microtus arvalis: 7: $\mathrm{M}_{1}$ izq.; Microtus duodecimcostatus: 8: $\mathrm{M}_{1}$ izq.; Arvicola aff. sapidus: 9: $\mathrm{M}_{1}$ izq.; Oryctolagus cuniculus: 10: Serie dentaria inferior izq.: $P_{3}-P_{4}$.

población (López Martínez, 1980 a) y uno de los más frecuentes en la población de dicha especie de Valdocarros (Sesé et al., 2011 a). El M ${ }^{3}$ presenta el PC similar al morfotipo $\mathrm{n}^{\circ} 1$ de la población de dicha especie de Áridos-1 (López Martínez, 1980 a), bien representado también en la población de Valdocarros (Sesé et al., 2011 a).

Discusión: La morfología de este material es la característica de Microtus brecciensis del Pleistoceno Medio. La evolución de Microtus brecciensis durante el Pleistoceno Medio conlleva algunas modificaciones morfológicas de los $\mathrm{M}_{1}$ principalmente (Ayarzagüena \& López Martínez, 1976) y un aumento gradual de la talla (Gil, 1997).

Con respecto a las poblaciones de Microtus brecciensis bien representadas en otros yacimientos de Madrid, la talla del $\mathbf{M}_{1}$ 
del Arenero de Arriaga es mayor que las de Áridos-1 (López Martínez, 1980 a) y Valdocarros (Sesé et al., 2011 a), superando las longitudes los valores máximos de éstas. También es de mayor talla que la de las poblaciones de dicha especie del yacimiento del Pleistoceno Medio de Ambrona (Sesé, 1986; Sesé y Soto, 2005), datado por ESR y U (Resonancia de Espín Electrónico y series de Uranio) en un mínimo de edad de unos 350 ka (Falguères et al., 2006), y de los niveles inferiores de Gran Dolina y los niveles del Complejo de Tres Simas de Atapuerca, y próxima a la del único ejemplar del nivel mas superior de Gran Dolina (TD 11) del Pleistoceno Medio casi final (Sesé y Gil, 1987; Gil, 1997) que ha sido datado por luminiscencia (OSL y IRSL) en $240 \pm 44$ ka BP (Berger et al., 2008; Rodríguez et al., 2011).

En cuanto a Microtus cabrerae THOMAS, 1906, especie que por evolución sucede a la anterior durante el Pleistoceno Superior (Ayarzagüena \& López Martínez, 1976; Cabrera-Millet et al., 1982; López Martínez, 2003 y 2009; Garrido-García \& Soriguer Escofet, 2012), aunque los valores de las longitudes de la población de Microtus brecciensis del Arenero de Arriaga están por encima de los valores medios de las poblaciones de Microtus cabrerae de los yacimientos del Pleistoceno Superior de PRERESA (Sesé et al., 2011 b) y del norte de Madrid de Pinilla del Valle (Toni \& Molero, 1990), en estas dos últimas poblaciones los valores máximos son mucho mayores $(\mathrm{L}=3,51$ y $\mathrm{L}=3,48$ respectivamente). Identificado este arvicolino inicialmente en Pinilla del Valle como Microtus brecciensis (Toni \& Molero, 1990), yacimiento al que dichos autores asignaban una edad del final del Pleistoceno Medio, fue posteriormente determinado como Microtus cabrerae y el yacimiento datado por TL en $90.961 \pm 7.881 \mathrm{BP}$, es decir, del Pleistoceno Superior (Arsuaga et al., 2010; Pérez González et al., 2010). La anchura de los $\mathrm{M}_{1}$, que es otro de los caracteres que utilizan Ayarzagüena \& López Martínez (1976) para distinguir ambas especies, en la población del Arenero de Arriaga alcanza valores mayores que los de las poblaciones de Microtus brecciensis de Áridos-1 (López Martínez, 1980 a) y Valdocarros (Sesé et al., 2011 a) y es menor que el valor máximo de Microtus cabrerae de PRERESA (Sesé et al., 2011 b) y HAT (Sesé et al., 2011 a), y menor también que los que son más frecuentes en poblaciones actuales según datos de Ayarzagüena \& López Martínez (1976).

Microtus brecciensis es una especie típica del Pleistoceno Medio de la Península Ibérica que fue muy común en la mayoría de los yacimientos de esta edad (Ayarzagüena \& López Martínez, 1976; Sesé, 1994; Sesé \& Sevilla, 1996; Arribas, 2004). La distribución de su descendiente, Microtus cabrerae, especie actualmente restringida a la Península Ibérica, está fragmentada en distintos puntos situados en el Prepirineo aragonés, Sistema Ibérico meridional, Sistema Central, Sierras Béticas, algunas áreas zamoranas y del sur de Portugal (Blanco, 1998; Palomo \& Gisbert, 2002).

\section{Microtus arvalis (PALLAS, 1978) - Topillo campesino (Fig. 1: 7)}

Material: 2 frs. de mandíbula izq. con $\mathrm{I}_{-} \mathrm{M}_{1} ; 2 \mathrm{M}_{1} ; 1$ fr. $\mathrm{M}_{1}$; $\mathrm{NMI}=3$

Medidas: $4 \mathrm{M}_{1}: \mathrm{L}=2,87-3,11 ; \mathrm{L}$ med. $=2,97 ; \mathrm{A}=1,08$ 1,26 ; A med. $=1,18$
Descripción: Molares arrizodontos, con cemento rellenando los ángulos entrantes. El $\mathrm{M}_{1}$ tiene lóbulo posterior, los cinco primeros triángulos cerrados, ángulos entrantes LRA 4 y BRA 3 alternos, T6 y T7 bien formados, alternos y confluyentes en la cúspide anterior redondeada u ovoide, LRA 5 y BRA 4 marcados. En uno de los cuatro $\mathrm{M}_{1}$ se desarrolla en la cúspide anterior un T9. El esmalte está diferenciado: es más grueso en el borde anterior de los triángulos, y falta en la parte mesial de la cúspide anterior.

Discusión: Esta morfología es similar a la de las poblaciones de Microtus arvalis del Pleistoceno Medio avanzando y final, que es cuando empieza a ser común su registro en la Península Ibérica (Sesé, 1994; Sesé \& Sevilla, 1996), Pleistoceno Superior, Holoceno y la actualidad. Es la primera vez que se cita esta especie en el Pleistoceno del sur de Madrid. Actualmente en España solo se encuentra en la mitad norte peninsular; la Comunidad de Madrid es una de la zonas del centro limítrofes de su distribución más meridional, aunque es más frecuente al norte de esta provincia (Palomo \& Gisbert, 2002).

\section{Microtus duodecimcostatus (SELYS-LONG- CHAMPS, 1839) - Topillo mediterráneo (Fig. 1: 8)}

Material y medidas: 1 fr. de mandíbula izq. con $\mathrm{M}_{1}: \mathrm{L}=$ 3,$07 ; \mathrm{A}=1,06 ; \mathrm{NMI}=1$

Descripción: $\mathrm{El} \mathrm{M}_{1}$, que tiene el lóbulo posterior fragmentado, presenta los tres primeros triángulos de esmalte cerrados, T4 y T5 alternos y confluyentes formando el característico rombo "pitimiano", cúspide anterior ovoide y en la que confluyen T6 y T7; los triángulos son asimétricos y los ángulos entrantes LRA 5 y BRA 4 no son muy profundos delimitando un cuello ancho.

Discusión: La morfología descrita es la característica de Microtus duodecimcostatus actual, similar a la de las poblaciones de la especie de los yacimientos del final del Pleistoceno Medio de Cueva del Agua (López Martínez y Ruiz Bustos, 1977) y del Pleistoceno Superior de PRERESA (Sesé et al., 2011 b), Pinilla del Valle (Toni \& Molero, 1990), y yacimientos franceses de Nestier, Lazaret y L'Hortus (Chaline, 1972).

El registro de esta especie empieza a ser frecuente en las asociaciones faunísticas del final del Pleistoceno Medio (Sesé, 1994; Sesé \& Sevilla, 1996). En el sur de Madrid Microtus duodecimcostatus se ha registrado también en el yacimiento del Pleistoceno Superior de PRERESA (Sesé et al., 2011 b) como se ha dicho anteriormente. Actualmente tiene un amplia distribución en la Península Ibérica excepto en la región noroccidental, gran parte de la región Cantábrica y algunas zonas del Pirineo oriental (Palomo \& Gisbert, 2002).

Arvicola aff. sapidus MILLER, 1908 - Rata de agua (Fig. 1: 9)

Material: $1 \mathrm{M}_{1} ; 1 \mathrm{M}_{3} ; 4 \mathrm{M}^{3}$ y 4 molares aislados de otro rango; $\mathrm{NMI}=2$

Medidas: $1 \mathrm{M}_{1}: \mathrm{L}=3,91 ; \mathrm{A}=1,66$

Descripción y discusión: Los molares hipsodontos, arrizodontos y con cemento rellenando los ángulos entrantes tienen 
la morfología y la talla características del género Arvicola. Aunque el $\mathrm{M}_{1}$ presenta cierto grado de desgaste, se aprecia que el esmalte está diferenciado siendo algo más grueso en la parte posterior de los triángulos, lo que es un carácter diferencial de Arvicola sapidus respecto de Arvicola terrestris (LINNAEUS, 1758) (López Martínez, 1980 a). Su talla es menor que la de las poblaciones actuales de Arvicola sapidus según datos de López Martínez (1980 a) y Sesé et al. (2011 b). Con respecto a las poblaciones mejor representadas de esta especie en los yacimientos del sur de Madrid, denominada Arvicola aff. sapidus por su menor talla con respecto a las poblaciones actuales de la especie, el ejemplar del Arenero de Arriaga entra dentro de la variación de la del yacimiento del Pleistoceno Medio de Áridos-1, algo superior a su valor medio (López Martínez, 1980 a), y está entre los valores mínimo y medio de las poblaciones de los yacimientos del Pleistoceno Superior de PRERESA (Sesé et al., 2011 b) y Pinilla del Valle (Toni \& Molero, 1990) que alcanzan valores máximos claramente mayores. La longitud de $\mathrm{M}_{1}$ es menor que la de los niveles superiores de Gran Dolina y los del Complejo Tres Simas de Atapuerca, excepto la de los niveles del final del Pleistoceno Medio de TN 4 y TN 5 que son similares (Gil, 1986).

La especie se registra en la Península Ibérica desde el Pleistoceno Medio avanzando (Sesé, 1994; Sesé \& Sevilla, 1996). Es endémica del sudoeste europeo: actualmente solo se encuentra en la Península Ibérica, en donde está ampliamente distribuida, y gran parte de Francia (Blanco, 1998; Palomo \& Gisbert, 2002).

Orden Lagomorpha BRANDT, 1855

Familia Leporidae FISCHER, 1817

Oryctolagus cuniculus (LINNAEUS, 1758)Conejo

(Fig. 1: 10)

Material: 2 frs. de mandíbula izq. con $\mathrm{P}_{3}-\mathrm{P}_{4} ; 1$ fr. de maxilar sup. con $\mathrm{I}^{1}-\mathrm{I}^{2}$ der. e $\mathrm{I}^{1}-\mathrm{I}^{2}$ izq.; 8 dientes de leche; $2 \mathrm{P}_{3} ; 8$ fr. $\mathrm{P}_{3}$; $4 \mathrm{P}^{2}$; 43 premolares y molares de otro rango; 6 incisivos. Hay también bastantes piezas esqueléticas, algunas de ellas enteras como las del carpo y tarso. $\mathrm{NMI}=6$

Medidas: $1 \mathrm{D}_{3}$ : $\mathrm{L}=1,91 ; \mathrm{A}=1,56 ; 3 \mathrm{P}_{3}: \mathrm{L}=2,22$ - 2,65 - 3,43; $\mathrm{A}=2,18-2,91-3,44 ; 2 \mathrm{P}^{2}: \mathrm{L}=1,29-1,44 ; \mathrm{A}=2,55-2,90$

Descripción y discusión: Los $\mathrm{P}_{3}$ muestran la talla y la morfología características de Oryctolagus cuniculus con anterofléxido ortogonal, ancho y de cierta profundidad, parafléxido marcado, hipofléxido poco rizado (López Martínez, 1989). La talla de la población del Arenero de Arriaga entra dentro de la variabilidad de las poblaciones de Oryctolagus cuniculus de los yacimientos del sur de Madrid del Pleistoceno Medio de Valdocarros (Sesé et al., 2011 a) y Pleistoceno Superior de PRERESA (Sesé et al., $2011 \mathrm{~b}$ ), aunque el valor máximo de la del Arenero de Arriaga excede a la de Valdocarros. Oryctolagus cf. lacosti del yacimiento del Pleistoceno Medio de Áridos-1 (López Martínez, 1980 a) es de talla mayor que la del Arenero de Arriaga. Dicha especie, según esta autora (o.c.), tiene una talla intermedia entre la de Lepus granatensis y Oryctolagus cuniculus algirus, por lo que es descartable para nuestro material, aunque en las publicaciones de Gamazo (1982) y Rus (1989) sobre el yacimiento del Arenero de Arriaga el lepórido aparecía citado como Oryctolagus cf. lacosti y Oryctolagus lacosti respectivamente.
Oryctolagus cuniculus tuvo su origen en España, expandiéndose al resto de Europa; su registro más antiguo conocido hasta ahora es en el yacimiento del Pleistoceno Medio inicial de Cúllar de Baza (López Martínez, 1989). En los yacimientos del sur de Madrid, el conejo es uno de los micromamíferos más abundantes tanto en el Pleistoceno Medio como en el Pleistoceno Superior (Sesé et al., 2011 a y b). Actualmente esta especie está ampliamente distribuida por toda la Península Ibérica (Blanco, 1998; Palomo \& Gisbert, 2002).

\section{Conclusiones}

\section{Consideraciones sobre la fauna}

La asociación de micromamíferos determinada en el presente trabajo es la siguiente:
Orden Soricomorpha GREGORY, 1910
Crocidura sp.
Talpa sp.

Orden Rodentia BODWICH, 1821

Eliomys quercinus quercinus (LINNAEUS, 1766)

Apodemus sp.

Microtus brecciensis (GIEBEL, 1847)

Microtus arvalis (PALLAS, 1978)

Microtus duodecimcostatus (SELYS-LONGCHAMPS, 1839)

Arvicola aff. sapidus MILLER, 1908

Orden Lagomorpha BRANDT, 1855

Oryctolagus cuniculus (LINNAEUS, 1758)

Todos estos taxones son comunes con el resto de los yacimientos del sur de Madrid: Áridos-1, Valdocarros, PRERESA y HAT excepto Microtus arvalis y Talpa sp. que es la primera vez que se citan en esta zona, y el primero también en el yacimiento del Arenero de Arriaga (véase Tabla 1). Sin embargo, en el Arenero de Arriaga no se registra Cricetulus (Allocricetus bursae), una especie que está presente en todos los mencionados yacimientos del Pleistoceno Medio y Superior excepto en HAT. Además, todos los taxones del Arenero de Arriaga habitan actualmente en la zona, excepto Microtus brecciensis, sustituida actualmente por su especie descendiente Microtus cabrerae, que también vive actualmente en Madrid y cuya distribución en la Península Ibérica está fragmentada tal como se ha dicho anteriormente. Microtus arvalis solo se encuentra actualmente en la mitad norte peninsular; la Comunidad de Madrid, es una de las zonas del centro peninsular limítrofes de su distribución más meridional. Los demás taxones del Arenero de Arriaga tienen por lo general una amplia distribución en toda la Península Ibérica. 
Tabla 1.-Taxones de micromamíferos de los yacimientos de los valles del Jarama y Manzanares de Madrid: Áridos-1 (López Martínez, 1980), Valdocarros y HAT (Sesé et al., 2011 a) y PRERESA (Sesé et al., 2011 b) y Arenero de Arriaga. Dataciones: de Áridos-1, Valdocarros y HAT (Panera et al., 2001) y de PRERESA (Sesé et al., 2011 b).

\begin{tabular}{|c|c|c|c|c|c|}
\hline TAXONES / YACIMIENTOS DE MADRID & ÁRIDOS-1 & VALDOCARROS & ARRIAGA & PRERESA & HAT \\
\hline SITUACIÓN GEOGRÁFICA & Jarama & Jarama & Manzanares & Manzanares & Jarama \\
\hline SITUACIÓN ESTRATIGRÁFICA & Arganda I & Arganda II & Arriaga I & & Arganda IV \\
\hline & \multicolumn{3}{|c|}{ Pleistoceno Medio } & \multicolumn{2}{|c|}{ Pleistoceno Superior } \\
\hline TAXONES / DATACIONES & $335-370 \mathrm{ka}$ & $255-269 \mathrm{ka}$ & & $84+-5,6 \mathrm{ka}$ & $74+16 /-12,1 \mathrm{ka}$ \\
\hline Erinaceus europaeus & & $\mathrm{x}$ & & $\mathrm{x}$ & \\
\hline Sorex sp. & $\mathrm{x}$ & & & & \\
\hline Crocidura russula $\mathrm{x}$ / C.aff. russula + & $\mathrm{x}$ & + & & $\mathrm{x}$ & \\
\hline Crocidura sp. & & & $\mathrm{x}$ & & \\
\hline Soricidae indet. & & & & & $\mathrm{x}$ \\
\hline Talpa sp. & & & $\mathrm{x}$ & & \\
\hline Eliomys quercinus & $\mathrm{x}$ & $\mathrm{x}$ & $\mathrm{x}$ & $\mathrm{x}$ & $\mathrm{x}$ \\
\hline Castor fiber & $\mathrm{x}$ & $\mathrm{x}$ & & & \\
\hline Apodemus cf. sylvaticus x / Apodemus sp. + & $\mathrm{x}$ & + & + & + & + \\
\hline Cricetulus (Allocricetus) bursae & $\mathrm{x}$ & $\mathrm{x}$ & & $\mathrm{x}$ & \\
\hline Microtus brecciensis & $\mathrm{x}$ & $\mathrm{x}$ & $\mathrm{x}$ & & \\
\hline Microtus cabrerae x / M. cf. cabrerae + & & & & $\mathrm{x}$ & + \\
\hline Microtus arvalis & & & $\mathrm{x}$ & & \\
\hline Microtus duodecimcostatus & & & $\mathrm{x}$ & $\mathrm{x}$ & \\
\hline Arvicola aff. sapidus & $\mathrm{x}$ & $\mathrm{x}$ & $\mathrm{x}$ & $\mathrm{x}$ & $\mathrm{x}$ \\
\hline Oryctolagus cf. lacosti & $\mathrm{x}$ & & & & \\
\hline Oryctolagus cuniculus & & $\mathrm{x}$ & $\mathrm{x}$ & $\mathrm{x}$ & \\
\hline cf. Oryctolagus sp. & & & & & $\mathrm{x}$ \\
\hline Pipistrellus sp. & $\mathrm{x}$ & & & & \\
\hline Vespertilionidae indet. & $\mathrm{x}$ & & & & \\
\hline Rhinolophus ferrumequinum & & & & $\mathrm{x}$ & \\
\hline
\end{tabular}

\section{Biocronología}

La asociación de micromamíferos del yacimiento del Arenero de Arriaga, por su composición y especialmente por el estadio evolutivo de Microtus brecciensis y Arvicola aff. sapidus, lo sitúan biostratigráficamente en una edad del final del Pleistoceno Medio. Asimismo, la asociación de macromamíferos es característica del Pleistoceno Medio, fundamentalmente por la presencia de Elephas (Palaeo- loxodon) antiquus (Sesé \& Soto, 2000 y 2002 a y b). Las dataciones realizadas recientemente de la base al techo en la secuencia en la que está situado el yacimiento del Arenero de Arriaga, han proporcionado unos valores entre fechas anteriores a 134133 ka BP para Arriaga I (nivel de donde proceden los micromamíferos) y Arriaga II, hasta $96 \pm 21 / 13$ ka BP para Arriaga III (Silva et al., 2011 y 2012), que confirman la edad propuesta a partir de la asociación faunística. 


\section{Tafonomía}

El material dentario y esquelético presenta en general muy buen estado de conservación. Hay algunas mandíbulas y maxilares superiores de casi todos los taxones identificados que conservan parte de la dentición, lo que indica que no han debido sufrir mucho arrastre ni rodamiento. En algunos dientes de arvicólidos y del conejo se observa la corrosión característica de la acción de la digestión, en general moderada, que parece indicar su procedencia por la acción de predación. La presencia de bastantes individuos juveniles de conejo, tal como sucede también en otros yacimientos del sur de Madrid, parece indicar también una actividad predadora (López Martínez, 1980 a y b; Sesé et al., 2011 a y b). La predominancia de un taxón, en este caso del conejo, es también característica de acumulaciones producidas por predadores. Todo ello hace suponer que al menos la mayor parte de la concentración de restos de micromamíferos se debe muy posiblemente a egagrópilas de aves rapaces.

\section{Paleoecología y Paleoclimatología}

Es notable en el yacimiento del Arenero de Arriaga el predominio de Oryctolagus cuniculus, cuyo porcentaje del NMI constituye casi la tercera parte del total de la fauna (30 \%) (véase Tabla 2). Esta abundancia del conejo ocurre también en otros yacimientos del sur de Madrid como Áridos-1, Valdocarros y PRERESA, tal como han puesto de manifiesto Sesé et al. (2011 b).

Teniendo en cuenta los requerimientos ambientales (según López Martínez, 1980 a; Sesé, 2005; Blanco, 1998; Palomo \& Gisbert, 2002) de los taxones que se registran en el yacimiento del Arenero de Arriaga, éstos se distribuyen en los siguientes biotopos: de bosque templado: Eliomys quercinus quercinus y Apodemus sp., de bordes de cursos de agua con vegetación ribereña: Arvicola aff. sapidus, de praderas muy húmedas con vegetación ribereña: Microtus brecciensis, de praderas húmedas con vegetación herbácea o arbustiva: Talpa sp., Microtus arvalis y Microtus duodecimcostatus, de praderas húmedas y secas con vegetación arbustiva: Oryctolagus cuniculus y de praderas secas con vegetación arbustiva: Crocidura sp. No hay registro de especies que podrían estar ligadas a zonas esteparias o secas y áridas como Cricetulus (Allocricetus) bursae, pre-
Tabla 2.-Abundancia de los taxones de micromamíferos del yacimiento del Arenero de Arriaga según el NMI y el porcentaje (\%) del NMI.

\begin{tabular}{|l|c|c|}
\hline \multicolumn{1}{|c|}{ TAXONES } & NMI & $\%$ NMI \\
\hline Crocidura sp. & 1 & 5 \\
\hline Talpa sp. & 1 & 5 \\
\hline Eliomys quercinus quercinus & 2 & 10 \\
\hline Apodemus sp. & 2 & 10 \\
\hline Microtus brecciensis & 2 & 10 \\
\hline Microtus arvalis & 3 & 15 \\
\hline Microtus duodecimcostatus & 1 & 5 \\
\hline Arvicola aff. sapidus & 2 & 10 \\
\hline Oryctolagus cuniculus & 6 & 30 \\
\hline & 20 & 100 \\
\hline
\end{tabular}

sente sin embargo en otros yacimientos del Pleistoceno Medio y Superior del sur de Madrid (véase Tabla 1). Por tanto, el paisaje general que indican los micromamíferos es fundamentalmente abierto $(80 \%)$, en el que predominarían las praderas muy húmedas y húmedas con bastante vegetación herbácea o arbustiva, y también de zonas que podrían ser menos húmedas y secas con vegetación arbustiva, pero también habría una buena representación de las zonas boscosas $(20 \%)$. Todo este conjunto, con bastante representación de taxones ligados a zonas húmedas $\mathrm{y}$, en general, con buen desarrollo de la vegetación, indica unos requerimientos de humedad ligadas evidentemente en buena parte a la proximidad del río, pero también a unas condiciones climáticas de cierta humedad ambiental que propiciarían la existencia de dicho entorno.

Eliomys quercinus quercinus y los géneros Apodemus y Crocidura son indicadores termófilos. Microtus brecciensis, Microtus duodecimcostatus y Oryctolagus cuniculus se consideran especies típicamente mediterráneas. La asociación de micromamíferos indica por tanto unas condiciones de clima templado.

La asociación faunística del Arenero de Arriaga es similar a otras del Pleistoceno Medio de la Península Ibérica (Sesé, 1994) y especialmente del sur de Madrid, tanto de esta edad como del Pleistoceno Superior (Sesé et al., 2011 b), claro exponente del carácter templado del clima que debió de predominar por lo general en la Meseta en esas épocas, bastante similar al actual. 


\section{AGRADECIMIENTOS}

Dedico este trabajo a Nieves López, con quien lo comencé pero que lamentablemente no ha podido ver finalizado por su temprana muerte. Nieves fue quien me inició en el estudio de los micromamíferos dirigiéndome la Tesis de Licenciatura primero y la Tesis Doctoral después. Tuve también la oportunidad de colaborar con ella en muchos yacimientos y trabajos como este, lo que siempre fue para mí una fuente continua de aprendizaje. Guardo recuerdos entrañables de nuestras salidas al campo para excavar y lavar sedimento del yacimiento de Arriaga en el embalse de las proximidades del arenero. Siempre le estaré agradecida por lo mucho que aprendí de ella y por la amistad que siempre me brindó.

Quiero expresar mi sincero agradecimiento al lamentablemente también fallecido Manolo Hoyos, que nos explicaba tan gráficamente y de forma tan sencilla e inteligible su interpretación de la geología y formación del yacimiento, y a Inmaculada Rus, directora de la excavación, que nos encargaron a Nieves López y a mi el estudio de los micromamíferos, a Paloma Sevilla, Ángeles Sacristán y Ester Herráez que amablemente nos ayudaron en el lavado del sedimento en el campo, a José Arroyo que realizó la delineación de los dibujos de los dientes de micromamíferos, a Enrique Soto que retocó los dibujos con un programa informático y realizó el montaje de la figura, y a Javier Murelaga y otro revisor anónimo cuyas sugerencias al manuscrito han contribuido a su mejora.

\section{Referencias}

Arribas, O. (2004). Fauna y paisaje de los Pirineos en la Era Glaciar. Lynx. ed., Barcelona: 540 pp.

Arsuaga, J.L.; Baquedano, B.; Pérez-González, A.; Sala, M.T. N.; García, N.; Álvarez-Lao, D.; Laplana, C.; Huguet, R.; Sevilla, P.; Maldonado, E.; Blain, H.-A.; Quam, R.; Ruiz Zapata, M.B.; Sala, P.; Gil García, M.J.; Uzquiano, P. \& Pantoja, A. (2010). El yacimiento arqueopaleontológico del Pleistoceno Superior de la Cueva del Camino en el Calvero de la Higuera (Pinilla del Valle, Madrid). In: Actas de la $1^{a}$ Reunión de científicos sobre cubiles de hiena (y otros grandes carnívoros) en los yacimientos arqueológicos de la Península Ibérica (Baquedano, E. \& Rosell, J., Dirs.). Zona Arqueológica, 13: 422-442

Ayarzagüena, J. \& López Martínez, N. (1976). Estudio filogenético y comparativo de Microtus cabrerae y Microtus brecciensis. Doñana Acta Vertebrata, 3 (2): 181-204.

Baena, J.; Conde, C.; Gamazo, M.; Sesé, C. \& Soto, E. (2002). Repertorio de yacimientos paleolíticos del Manzanares y del Jarama. In: Bifaces y Elefantes. La investigación del Paleolitico Inferior en Madrid. (Panera Gallego, J. \& Rubio Jara, S., Eds.). Zona Arqueológica, 1: 460-491.

Baena, J.; Baquedano, I.; Beneítez, P.; Cañaveras, J.C.; Carrión, E.; Lario, J.; Martín-Escorza, C.; Mazo, A.V.; Millán, A.; Sánchez, S.; Sanz, E.; Sesé, C.; Silva, P.G. \& Yravedra, J. (2010). Síntesis: Interpretación general del yacimiento de TAFESA (Madrid). In: Estudio del yacimiento del Pleistoceno madrileño de TAFESA (antigua TRANSFESA). (Baena Preysler, J \& Baquedano, I. Coords.). Zona Arqueológica, 14: 189-202.

Berger, G.W.; Pérez-González, A.; Carbonell, E.; Arsuaga, J.L.; Bermúdez de Castro, J.M. \& Ku, T.L. (2008). Luminescence chronology of cave sediments at the Atapuerca paleoanthropological site, Spain. Journal of Human Evolution, 55: 300-311. doi:10.1016/j.jhevol.2008.02.012

Blanco, J.C. (1998): Mamíferos de España: I: Insectívoros, Quirópteros, Primates y Carnívoros de las Península Ibérica, Baleares y Canarias y II: Cetáceos, Artiodáctilos, Roedores y Lagomorfos de las Península Ibérica, Baleares y Canarias. Geoplaneta, Barcelona: 457 y 383 pp.

Cabrera-Millet, M.; López Martínez, N. \& Michaux, J. (1982). Un exemple de lignee endemique iberoccitane, les campagnols Microtus brecciensis et Microtus cabrerae (Mammalia, Rodentia): Etude phylogenetique et contexte ecologique d'un phenomene evolutif recent. In: Actes du symposium páleontologique G. Cuvier (Buffetaut, E., Mazin, J.M. \& Salmon, E., Eds.). Montbeliard: 69-83.

Chaline, J. (1972). Les Rongeurs du Pléistocène Moyen et Supérieur de France. (Systématique, Biostratigraphie, Paléoclimatologie). Cahiers de Paleontologie, C.N.R.S.: 410 pp.

Domínguez Alonso, R.M.; Arcos Fernández S.; Ruiz Zapata, B. \& Gil García, M.J. (2009). Nuevos datos sobre la Terraza Compleja del Butarque en Villaverde Bajo. Actas de las Cuartas Jornadas de Patrimonio Arqueológico en la Comunidad de Madrid, Alcalá de Henares, 2007: 339-343.

Falguères, C.; Bahain, J.J.; Pérez-González, A.; Mercier ; N.; Santonja, M. \& Dolo, J.-M. (2006). The Lower Acheulian site of Ambrona, Soria (Spain): ages derived from a combined ESR/U-series model. Journal of Archaeological Science, 33: 149-157. doi:10.1016/ j.jas.2005.07.006

Gamazo, M. (1982). Prospecciones en las terrazas de la margen derecha del río Manzanares (Getafe y RibasVaciamadrid). Noticiario Arqueológico Hispánico, 14: 9-148.

Garrido-García, J.A. \& Soriguer-Escofet, R.C. (2012). Cabrera's Vole Microtus cabrerae Thomas, 1906 and the subgenus Iberomys during the Quaternary: Evolutionary implications and conservation. Geobios, 45: 437-444. doi:10.1016/j.geobios.2011.10.014

Gil, E. (1986). Taxonomía y bioestratigrafía de Micromamíferos del Pleistoceno Medio, especialmente Roedores, de los rellenos kársticos de la Trinchera de ferrocarril de la Sierra de Atapuerca (Burgos). Tesis Doctoral, Universidad de Zaragoza: 229 pp.

Gil, E. (1997). Bioestratigrafía y evolución de Microtus brecciensis en los yacimientos del Pleistoceno de Atapuerca (Burgos, España). Geogaceta, 21: 113-116.

Goy, J.L.; Pérez-González, A. \& Zazo, C., 1989. Cartografía y Memoria del Cuaternario y Geomorfología, Hoja de Madrid (559). Mapa Geológico de España. 
Escala 1:50.000. 2a Serie (MAGNA). Instituto Tecnológico GeoMinero de España. Servicio de Publicaciones del Ministerio de Industria, Madrid.

López Martínez, N. (1980 a). Los micromamíferos (Rodentia, Insectivora, Lagomorpha Chiroptera), del sitio de ocupación Achelense de Áridos-1 (Arganda, Madrid). In: Ocupaciones Achelenses en el Valle del Jarama (Arganda, Madrid) (Santonja, M.; López Martínez, N. \& Pérez-González, A., Eds.). Arqueología y Paleoecología, 1. Diputación Provincial de Madrid: 161-202.

López Martínez, N. (1980b). Análisis tafonómico y paleoecológico de los vertebrados de Áridos-1. (Pleistoceno Medio, Arganda, Madrid). In: Ocupaciones Achelenses en el Valle del Jarama (Arganda, Madrid) (Santonja, M.; López Martínez, N. \& Pérez-González, A., Eds.). Arqueología y Paleoecología, 1. Diputación Provincial de Madrid: 307-320.

López Martínez, N. (1989). Revisión sistemática y biostratigráfica de los lagomorfos (Mammalia) del Terciario y Cuaternario de España. Memorias del Museo Paleontológico de la Universidad de Zaragoza, 3 (3): 343 pp.

López Martínez, N. (2003). La búsqueda del centro de origen en biogeografía histórica. Graellsia, 59: 503-522.

López Martínez, N. (2009). Time asymmetry in the paleobiogeography history of species. Bulletin de la Société Géologique de France, 180 (1): 45-55.

López Martínez, N. \& Ruiz Bustos, A. (1977). Descubrimiento de dos yacimientos del

Pleistoceno Medio en el karst de la Sierra Alfaguara (Granada). Síntesis estratigráfica de este período en la región Bética. Estudios Geológicos, 33 : 255-265.

Mazo, A.V. (2010). Los macroverterbados del Pleistoceno Medio del yacimiento de TAFESA (Madrid). In: Estudio del yacimiento del Pleistoceno madrileño de TAFESA (antigua TRANSFESA). (Baena Preysler, J \& Baquedano, I. Coords.). Zona Arqueológica, 14:141152.

Meulen, A.J. Van der (1973). Middle Pleistocene smaller Mammals from the Monte Peglia (Orvieto, Italy) with special reference to the phylogeny of Microtus (Arvicolidae, Rodentia). Quaternaria, 17: 144 pp.

Michaux, J. \& Pasquier, L. (1974). Dynamique des populations de Mulots (Rodentia, Apodemus) en Europe durant le Quaternaire. Premières données. Bulletin de la Société Géologique de France, 16, 4 : 431-439.

Palomo, J. \& Gisbert, J. (Eds.) (2002): Atlas de los Mamíferos Terrestres de España. Dirección General de Conservación de la Naturaleza-SECEM-SECEMU, Madrid: 564 pp.

Panera, J.; Pérez-González, A.; Rubio-Jara, S. \& Sesé, C. (2005). El yacimiento paleolítico de HAT en el valle del Jarama: una aportación del Cuaternario de la cuenca de Madrid al debate sobre el inicio del Paleolítico medio. In: Geoarqueología y Patrimonio en la Península Ibérica y el entorno Mediterráneo (Santonja, M.; Pérez-González, A. \& Machado, M.J., Eds.). ADEMA, Soria: 251-260.
Panera, J.; Torres, T.; Pérez-González, A.; Ortiz, J.E.; Rubio-Jara, S. \& Uribelarrea, D. (2011). Geocronología de la Terraza Compleja de Arganda en el valle del río Jarama (Madrid, España). Estudios Geológicos, 67 (2): 495-504.

Pasquier, L. (1974). Dynamique evolutive d'un sousgenre de Muridae, Apodemus (Sylvaemus). Etude biométrique des caractéres dentaires de populations fossiles et actuelles d'Europe occidentale. Tesis Doctoral, Université des Sciences et Techniques du Languedoc, Montpellier: 184 pp.

Pérez-González, A. (1971). Estudio de los procesos de hundimiento en el valle del río Jarama y sus terrazas (nota preliminar). Estudios Geológicos, 27 (4): 317324.

Pérez-González, A. (1980). Geología y estratigrafía de los yacimientos de Áridos en la llanura aluvial de Arganda (Madrid). In: Ocupaciones Achelenses en el Valle del Jarama (Arganda, Madrid) (Santonja, M.; López Martínez, N. \& Pérez-González, A., Eds.). Arqueología y Paleoecología, 1. Diputación Provincial de Madrid: 49-61.

Pérez-González, A.; Rubio Jara, S.; Panera, J. \& Uribelarrea, D. (2008). Geocronología de la sucesión arqueoestratigráfica de Los Estragales en la Terraza Compleja de Butarque (Valle del río Manzanares, Madrid). Geogaceta, 45: 39-42.

Pérez-González, A.; Karampaglidis, T.; Arsuaga, J.L.; Baquedano, E.; Bárez, S.; Gómez, J.J.; Panera, J.; Márquez, B.; Laplana, C.; Mosquera, M.; Huguet, R.; Sala, P.; Arriaza, M.C.; Benito, A. \& Maldonado, E. (2010). Aproximación geomorfológica a los yacimientos del Pleistoceno Superior del Calvero de la Higuera en el Valle Alto del Lozoya (Sistema Central Español, Madrid). In: Actas de la $1^{a}$ Reunión de Científicos sobre cubiles de hienas (y otros grandes carnívoros) en los yacimientos arqueológicos de la Península Ibérica (Baquedano, E. \& Rosell, J., Eds.). Zona Arqueológica, 13: 404-419.

Rodríguez, J.; Burjachs, F.; Cuenca-Bescós, G.; García, N.; Van der Made, J.; Pérez González, A.; Blain, H.A.; Expósito, I.; López-García, J.M.; García Antón, M.; Allué, E.; Cáceres, I.; Huguet, R.; Mosquera, M.; Ollé, A.; Rosell, J.; Parés, J.M.; Rodríguez, X.P.; Díez, C.; Rofes, J.; Sala, R.; Saladié, P.; Vallverdú, J.; Bennasar, M.L.; Blasco, R.; Bermúdez de Castro, J.M. \& Carbonell, E., (2011): One million years of cultural evolution in a stable environment at Atapuerca (Burgos, Spain). Quaternary Science Reviews, 30 (11-12): 1396-1412.

Rus, I. (1983). Más de un siglo de estudios. El Paleolítico en el Valle del Manzanares. Revista de Arqueología, Año 4, Segunda Época, 32: 6-15.

Rus, I. (1989). El Paleolítico inferior en el valle del Manzanares. Raña, 7: 33·34.

Rus, I. \& Vega, L.G. (1984). El yacimiento de Arriaga II: problemas de una definición actual de los suelos de ocupación. Primeras Jornadas de Metodología de Investigación Prehistórica, Soria, 1981: 387-404.

Rus, I. \& Enamorado, J. (1991). Flint supply in the Manzanares valley: the acheulean site of Arriaga (Madrid, 
Spain). VI Flint International Symposium, Granada: 569-576.

Sanchiz, B. (1991). Algunas herpetofaunas de yacimientos del Pleistoceno Medio Ibérico. Revista Española de Herpetología, 5: 9-13.

Sesé, C. (1986). Insectívoros, roedores y lagomorfos (Mammalia) del sitio de ocupación achelense de Ambrona (Soria, España). Estudios Geológicos, 42: 355-359.

Sesé, C. (1994). Paleoclimatical interpretation of the Quaternary small mammals of Spain. Geobios, 27, 6: 753-767.

Sesé, C. (2005). Aportación de los micromamíferos al conocimiento paleoambiental del Pleistoceno Superior de la Región Cantábrica: Nuevos datos y síntesis. In: Neandertales Cantábricos, estado de la cuestión (Montes Barquín, R. \& Lasheras Corruchaga, J.A., Eds.). Monografias del Museo Nacional y Centro de Investigación de Altamira, 20: 167-200.

Sesé, C. (2010). Micromamíferos del yacimiento del Pleistoceno Medio de TAFESA (Madrid). Las huellas de nuestro pasado. In: Estudio del yacimiento del Pleistoceno madrileño de TAFESA (antigua TRANSFESA). (Baena Preysler, J \& Baquedano, I. Coords.). Zona Arqueológica, 14: 135-139.

Sesé, C. \& Gil, E. (1987). Los Micromamíferos del Pleistoceno Medio del complejo cárstico de Atapuerca (Burgos). In: El Hombre fósil de Ibeas y el Pleistoceno de la Sierra de Atapuerca. I. (Aguirre, E.; Carbonell, E. \& Bermúdez De Castro, J.M., Eds.). Junta de Castilla y León, Consejería de Cultura y Bienestar Social: 75-88.

Sesé, C. \& Sevilla, P. (1996). Los micromamíferos del Cuaternario peninsular español: Cronoestratigrafía e implicaciones bioestratigráficas. Revista Española de Paleontología, $\mathrm{N}^{\circ}$ Extraordinario: 278-287.

Sesé, C. \& Soto, E. (2000). Vertebrados del Pleistoceno de Madrid. In: El patrimonio paleontológico de la Comunidad de Madrid. Floras y Faunas. (Morales, J.; Nieto, M.; Amezua, L.; Fraile, S.; Gómez, E.; Herráez, E.; Peláez-Campomanes, P., Salesa, M.J.; Sánchez, I.M. \& Soria, D., Eds.). Arqueología, Paleontología y Etnografia, 6: 216-243.

Sesé, C. \& Soto, E. (2002 a). Vertebrados del Pleistoceno del Jarama y Manzanares. In: Bifaces y Elefantes. La investigación del Paleolítico Inferior en Madrid. (Panera Gallego, J. \& Rubio Jara, S., Eds.). Zona Arqueológica, 1: 318-337.

Sesé, C. \& Soto, E. (2002 b). Catálogo de los yacimientos de Vertebrados del Pleistoceno en las terrazas de los ríos Jarama y Manzanares. In: Bifaces y Elefantes.
La investigación del Paleolítico Inferior en Madrid. (Panera Gallego, J. \& Rubio Jara, S., Eds.). Zona Arqueológica, 1: 430-457.

Sesé, C. \& Soto, E. (2005). Mamíferos del Yacimiento del Pleistoceno Medio de Ambrona: análisis Faunístico e interpretación Paleoambiental. In: Los yacimientos Paleolíticos de Ambrona y Torralba (Soria). Un siglo de investigación arqueológica. (Santonja, M. \& Pérez González, A., Eds.). Zona Arqueológica, 5: 258-280.

Sesé, C.; Panera, J.; Rubio-Jara, S. \& Pérez-González, A. (2011 a). Micromamíferos del Pleistoceno Medio y Pleistoceno Superior en el Valle del Jarama: yacimientos de Valdocarros y HAT (Madrid, España). Estudios Geológicos, 67 (1): 131-151.

Sesé, C.; Rubio-Jara, S.; Panera, J. \& Pérez-González, A. (2011 b). Micromamíferos del Pleistoceno Superior del yacimiento de PRERESA en el valle del Manzanares y su contribución a la reconstrucción paleoambiental de la cuenca de Madrid durante el Pleistoceno. Estudios Geológicos, 67 (2): 471-494.

Silva, P.G. (2003). El Cuaternario del Valle inferior del Manzanares. Estudios Geológicos, 59: 107-131. doi:10.3989/egeol.03591-488

Silva, P.G.; López-Recio, M.; González Hernández, F.M.; Tapias, F.; Alarcón, A.; Cuartero, F.; Expósito, A.; Lázaro, I.; Manzano, I.; Martín, D.; Morín, J. \& Yravedra, J. (2008). Datos geoarqueológicos de la terraza compleja del Manzanares entre el sector del 12 de octubre y la desembocadura del arroyo Butarque (Villaverde, Madrid). Cuaternario y Geomorfología, 22 (3-4): 47-70. doi:10.3989/egeol.40338.134

Silva, P.G.; Tapias, F.; López-Recio, M.; Carrasco, P.; Morín, J.; Roquero, E. \& I. Rus (2011). Análisis estratigráfico del Arenero de Arriaga (Terraza Compleja del Manzanares, Madrid). Resúmenes XIII Reunión Nacional del Cuaternario, Andorra, 2011.

Silva, P.G.; López-Recio, M.; Cuartero, F.; Baena, J.; Manzano, I.; Martín, D.; Morín, J. \& Roquero, E. (2012). Contexto geomorfológico y principales rasgos tecnológicos de nuevos yacimientos del Pleistoceno Medio y Superior en el Valle Inferior del Manzanares (Madrid, España). Estudios Geológicos, 68 (1): 57-89.

Toni, I. \& Molero, G. (1990). Los roedores (Rodentia, Mammalia) del yacimiento cuaternario de Pinilla del Valle (Madrid). Actas de las IV Jornadas de Paleontología, Salamanca, 1988: 359-373.

Recibido el 13 de febrero de 2013 Aceptado el 23 de mayo de 2013 